\title{
Unified theory for certain generalized types of closed sets and some separation axioms
}

\author{
Bishwambhar Roy \\ Department of Mathematics \\ Women's Christian College, India \\ email: bishwambhar_roy@yahoo.co.in
}

\begin{abstract}
In this paper the notion of $\mu v \mathrm{~g}$-closed sets and certain characterizations of such sets have been given. As an application of $\mu \nu \mathrm{g}$ closed sets, the notion of $(\mu, v)$-regular spaces and $(\mu, v)$-normal spaces have been introduced and some characterizations of such spaces are also given.
\end{abstract}

\section{Introduction}

For the last one decade or so, a new area of study has emerged and has been rapidly growing. The area is concerned with the investigations of generalized topological spaces and several classes of generalized types of open sets. Recently, a significant contribution to the theory of generalized open sets, was extended by A. Császár [1, 2, 3]. It is observed that a large number of papers are devoted to the study of generalized open sets, containing the class of open sets and possessing properties more or less similar to those of open sets.

We recall some notions defined in [2]. Let $X$ be a non-empty set and $\operatorname{expX}$ denote the power set of $X$. We call a class $\mu \subseteq \operatorname{expX}$ a generalized topology [2], (briefly, GT) if $\varnothing \in \mu$ and union of elements of $\mu$ belong to $\mu$. A set $X$ with a GT $\mu$ on it is called a generalized topological space (briefly, GTS) and is denoted by $(X, \mu)$. For a GTS $(X, \mu)$, the elements of $\mu$ are called $\mu$-open 
sets and the complements of $\mu$-open sets are called $\mu$-closed sets. For $A \subseteq X$, we denote by $c_{\mu}(A)$ the intersection of all $\mu$-closed sets containing $A$, i.e., the smallest $\mu$-closed set containing $A$; and by $i_{\mu}(A)$ the union of all $\mu$-open sets contained in $A$, i.e., the largest $\mu$-open set contained in $A$ (see $[2,3])$.

It is easy to observe that $i_{\mu}$ and $c_{\mu}$ are idempotent and monotonic, where the operator $\gamma: \operatorname{expX} \rightarrow \operatorname{expX}$ is said to be idempotent if $A \subseteq X$ implies $\gamma(\gamma(A))$ $=\gamma(A)$ and monotonic if $A \subseteq B \subseteq X$ implies $\gamma(A) \subseteq \gamma(B)$. It is known from $[3,1]$ that if $\mu$ is a GT on $X, x \in X$ and $A \subseteq X$, then $x \in \mathcal{c}_{\mu}(A)$ iff $x \in M \in \mu$ $\Rightarrow M \cap A \neq \varnothing$. It is also well known from $[3,1]$ that $x \in i_{\mu}(A)$ if and only if there exists $\mathrm{U} \in \mu$ with $x \in \mathrm{U}$ such that $x \in \mathrm{U} \subseteq A$ and $\mathrm{c}_{\mu}(X \backslash A)=X \backslash i_{\mu}(A)$ and $i_{\mu}(X \backslash A)=X \backslash c_{\mu}(A)$.

\section{$2 \mu \nu g$-closed sets and $\mu \nu g$-open sets}

Definition 1 Let $\mu$ and $\nu$ be two GT's on a set $\mathrm{X}$. Then $\mathrm{A} \subseteq \mathrm{X}$ is called $\mu v \mathrm{~g}$-closed [4] if $\mathrm{c}_{v}(\mathrm{~A}) \subseteq \mathrm{U}$ whenever $\mathrm{A} \subseteq \mathrm{U}$ and $\mathrm{U} \in \mu$. The complement of $a \mu v \mathrm{~g}$-closed set is called a $\mu \mathrm{vg-open} \mathrm{set.}$

Proposition 1 Let $\mu$ and $\nu$ be two GT's on a set $\mathrm{X}$. Then for $\mathrm{A}, \mathrm{B} \subseteq \mathrm{X}$ the following holds:

(i) If $\mathrm{A}$ is $v$-closed then $\mathrm{A}$ is $\mu \mathrm{vg}$-closed.

(ii) If $\mathrm{A}$ is $\mu v \mathrm{v}$-closed and $\mu$-open then $\mathrm{A}$ is $v$-closed.

(iii) If $\mathrm{A}$ is $\mu \nu \mathrm{g}$-closed and $\mathrm{A} \subseteq \mathrm{B} \subseteq \mathrm{c}_{v}(\mathrm{~A})$, then $\mathrm{B}$ is $\mu \nu \mathrm{v}$-closed.

Proof. (i) Let $A$ be a $v$-closed subset of $X$ and $A \subseteq U \in \mu$. Then $c_{v}(A)=$ $A \subseteq U$ and thus $A$ is $\mu v$ g-closed.

(ii) Let $A$ be a $\mu \nu$ g-closed, $\mu$-open subset of $X$. Then $c_{v}(A) \subseteq A$ and hence $A$ is $v$-closed.

(iii) Let $\mathrm{B} \subseteq \mathrm{U}$ where $\mathrm{U}$ is a $\mu$-open set. Then $\mathrm{A} \subseteq \mathrm{U}$ and hence by $\mu v \mathrm{~g}$ closedness of $\bar{A}, c_{v}(A) \subseteq U$. Now $c_{v}(A) \subseteq c_{v}(B) \subseteq c_{v}\left(c_{v}(A)\right)=c_{v}(A)$. Hence $c_{v}(A)=c_{v}(B)$. Therefore $c_{v}(B) \subseteq U$ and hence B is $\mu v g$-closed.

Theorem 1 Let $\mu$ and $\nu$ be two GT's on $\mathrm{X}$. Then $\mathrm{A} \subseteq \mathrm{X}$ is $\mu \vee \mathrm{v}$-closed if and only if $\mathrm{c}_{v}(\mathrm{~A}) \cap \mathrm{F}=\varnothing$ whenever $\mathrm{A} \cap \mathrm{F}=\varnothing$ and $\mathrm{F}$ is $\mu$-closed.

Proof. Let $A$ be a $\mu \nu$ g-closed subset of $X$ and $F$ be $\mu$-closed with $A \cap F=\varnothing$. Then $A \subseteq X \backslash F$ where $X \backslash F$ is $\mu$-open. Thus $c_{v}(A) \subseteq X \backslash F$. Therefore we have $c_{v}(A) \cap F=\varnothing$. 
Conversely, let $A \subseteq \mathrm{U}$ and $\mathrm{U}$ be $\mu$-open. Then $A \cap(X \backslash \mathrm{U})=\varnothing$ where $\mathrm{X} \backslash \mathrm{U}$ is $\mu$-closed. Thus by hypothesis, $c_{v}(A) \cap(X \backslash U)=\varnothing$ and hence $c_{v}(A) \subseteq U$ showing $A$ to be $\mu v g$-closed.

Definition $2[5]$ Let $(X, \mu)$ be a GTS and $\mathrm{A} \subseteq \mathrm{X}$. Then the subset $\bigwedge_{\mu}(\mathrm{A})$ is defined by

$$
\bigwedge_{\mu}(A)=\left\{\begin{array}{l}
\bigcap\{\mathrm{G}: \mathrm{A} \subseteq \mathrm{G}, \mathrm{G} \in \mu\}, \text { if there exists } \mathrm{G} \in \mu \text { such that } \mathrm{A} \subseteq \mathrm{G} ; \\
\mathrm{X}, \text { otherwise. }
\end{array}\right.
$$

Theorem 2 Let $\mu$ and $\nu$ be two GT's on $\mathrm{X}$. Then $\mathrm{A}(\subseteq \mathrm{X})$ is $\mu \nu \mathrm{g}$-closed if and only if $\mathrm{c}_{\nu}(\mathrm{A}) \subseteq \bigwedge_{\mu}(\mathrm{A})$.

Proof. Suppose that $A$ is $\mu \nu$ g-closed. Let $x \notin \bigwedge_{\mu}(A)$. Then there exists a $\mu$-open set $\mathrm{G}$ with $\chi \notin \mathrm{G}$ and $A \subseteq \mathrm{G}$. Then $\chi \notin \mathrm{c}_{v}(A)$ (as $A$ is $\mu \nu$-closed). Thus $c_{v}(A) \subseteq \bigwedge_{\mu}(A)$.

Conversely, suppose that $c_{v}(A) \subseteq \bigwedge_{\mu}(A)$. Let $A \subseteq U$ where $U$ is $\mu$-open. Then $c_{v}(A) \subseteq \bigwedge_{\mu}(A) \subseteq \bigwedge_{\mu}(U)=U$. Thus $A$ is $\mu v$ g-closed.

Theorem 3 Let $\mu$ and $\vee$ be two GT's on $\mathrm{X}$. Then $\mathrm{A}(\subseteq \mathrm{X})$ is called $\mu \vee \mathrm{v}$-closed if and only if $\mathrm{c}_{\mu}(\{x\}) \cap A \neq \varnothing$ for each $\mathrm{x} \in \mathrm{c}_{\nu}(A)$.

Proof. Suppose that $A$ is $\mu \nu g$-closed and $c_{\mu}(\{x\}) \cap A=\varnothing$ for some $x \in c_{v}(A)$. Then $A \subseteq X \backslash c_{\mu}(\{x\})$ where $X \backslash c_{\mu}(\{x\})$ is $\mu$-open. Thus $c_{v}(A) \subseteq X \backslash c_{\mu}(\{x\}) \subseteq$ $X \backslash\{x\}$. This contradicts the fact that $x \in c_{v}(A)$.

Conversely, suppose that $A$ be not $\mu \nu$ g-closed. Then $c_{v}(A) \backslash U \neq \varnothing$ for some $\mu$-open set $U$ with $A \subseteq U$. Let $x \in c_{v}(A) \backslash U$. Then $x \in c_{v}(A)$ and $x \notin U$. Then $\mathrm{c}_{\mu}(\{x\}) \cap \mathrm{U}=\varnothing$ and hence $\mathrm{c}_{\mu}(\{x\}) \cap A \subseteq \mathrm{c}_{\mu}(\{x\}) \cap \mathrm{U}=\varnothing$. This shows that $c_{\mu}(\{x\}) \cap A=\varnothing$ for some $x \in c_{v}(A)$.

Theorem 4 Let $\mu$ and $\nu$ be two GT's on $\mathrm{X}$. Then a subset $\mathrm{A}(\subseteq \mathrm{X})$ is $\mu \mathrm{vg}$ open if and only if $\mathrm{F} \subseteq \mathfrak{i}_{v}(\mathrm{~A})$ whenever $\mathrm{F} \subseteq \mathrm{A}$ and $\mathrm{F}$ is $\mu$-closed.

Proof. Suppose that $A$ is $\mu \nu$ g-open. Let $F \subseteq A$ and $F$ be $\mu$-closed. Then $X \backslash A \subseteq X \backslash F \in \mu$ and $X \backslash A$ is $\mu v g$-closed. Thus $X \backslash i_{v}(A)=c_{v}(X \backslash A) \subseteq X \backslash F$ and hence $F \subseteq i_{v}(A)$.

Conversely, let $X \backslash A \subseteq U$ where $U$ is $\mu$-open. Then $X \backslash U \subseteq A$ and $X \backslash U$ is $\mu$-closed. Thus by the hypothesis, $X \backslash U \subseteq i_{v}(A)$ and thus $c_{v}(X \backslash A)=$ $X \backslash i_{v}(A) \subseteq U$. Hence $A$ is $\mu \nu g$-open. 
Definition 3 Let $\mu$ and $\nu$ be two GT's on $\mathrm{X}$. Then $\mu$ and $\nu$ is said to have the property $(*)$ if $\mathrm{A} \in \mu, \mathrm{B} \in v$ implies that $\mathrm{A} \cup \mathrm{B} \in \mu$.

Theorem 5 Let $\mu$ and $\nu$ be two GT's on $\mathrm{X}$ satisfying the property $(*)$. Then the following are equivalent:

(1) A is $\mu v g$-closed.

(2) $\mathfrak{c}_{v}(A) \backslash A$ does not contain any non-empty $\mu$-closed set.

(3) $\mathrm{c}_{v}(\mathrm{~A}) \backslash \mathrm{A}$ is $\mu \nu \mathrm{g}$-open.

Proof. $(1) \Rightarrow(2)$ : Suppose that $A$ is a $\mu v g$-closed set. Let $F \subseteq c_{v}(A) \backslash A$ and $\mathrm{F}$ be $\mu$-closed. Then $A \subseteq X \backslash F$ where $X \backslash F$ is $\mu$-open and hence, $c_{v}(A) \subseteq X \backslash F$. Therefore, we have $F \subseteq X \backslash c_{v}(A)$ and hence, $F \subseteq c_{v}(A) \cap\left(X \backslash c_{v}(A)\right)=\varnothing$. Thus $\mathrm{F}=\varnothing$.

$(2) \Rightarrow(3)$ : Let us assume that $\mathrm{F} \subseteq \mathrm{c}_{v}(A) \backslash A$ and $\mathrm{F}$ be $\mu$-closed. By (2), we have $\mathrm{F}=\varnothing$ and $\mathrm{F} \subseteq \mathrm{i}_{v}\left[\mathrm{c}_{v}(A) \backslash A\right]$. Hence by Theorem $4, \mathrm{c}_{v}(A) \backslash A$ is $\mu v g$-open.

$(3) \Rightarrow(1)$ : Suppose that $A \subseteq U$ and $U$ is $\mu$-open. Then, $c_{v}(A) \backslash U \subseteq c_{v}(A) \backslash A$. By $(3), c_{v}(A) \backslash A$ is $\mu \nu g$-open. Since $\mu$ and $v$ have the property $(*), c_{v}(A) \backslash U$

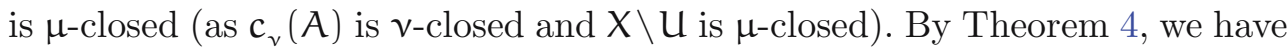
$c_{v}(A) \backslash U \subseteq i_{v}\left(c_{v}(A) \backslash A\right)=\varnothing$. [In fact if $i_{v}\left(c_{v}(A) \backslash A\right) \neq \varnothing$, then there exists some $x \in i_{v}\left(c_{v}(A) \backslash A\right)$. Then, there exists $G \in v$ such that $x \in G \subseteq c_{v}(A) \backslash A$. Since $G \subseteq X \backslash A$, we have $G \cap A=\varnothing$ and $G \in v$. Thus $G \cap c_{v}(A)=\varnothing$ and $G \subseteq X \backslash c_{v}(A)$. Therefore, we obtain $G \subseteq c_{v}(A) \cap\left(X \backslash c_{v}(A)\right)=\varnothing$.] Therefore, we have $c_{v}(A) \subseteq U$ and hence $A$ is $\mu v g$-closed.

Theorem 6 Let $\mu$ and $v$ be two GT's on $\mathrm{X}$ satisfying the property (*). A subset $\mathrm{A}$ of $\mathrm{X}$ is $\mu \mathrm{vg-open}$ if and only if $\mathrm{G}=\mathrm{X}$ whenever $\mathrm{G}$ is $\mu$-open and $i_{v}(A) \cup(X \backslash A) \subseteq G$.

Proof. Let $A$ be a $\mu \nu$ g-open set and G be $\mu$-open with $i_{v}(A) \cup(X \backslash A) \subseteq G$. Then $X \backslash G \subseteq c_{v}(X \backslash A) \backslash(X \backslash A)$. Since $X \backslash A$ is $\mu v g$-closed and $X \backslash G$ is $\mu$-closed, by Theorem $5, X \backslash G=\varnothing$ and hence $G=X$.

Conversely let us assume that $F \subseteq A$ and $F$ be $\mu$-closed. Since $\mu$ and $\nu$ have the property $(*)$, we have $i_{v}(A) \cup(X \backslash A) \subseteq i_{v}(A) \cup(X \backslash F)$ and $i_{v}(A) \cup(X \backslash F)$ is $\mu$-open. Thus by the hypothesis, $X=i_{v}(A) \cup(X \backslash F)$. Hence, $F=F \cap\left(i_{v}(A) \cup(X)\right.$ $F))=F \cap i_{v}(A) \subseteq i_{v}(A)$. Thus from Theorem 4 it follows that $A$ is $\mu v g$-open.

Theorem 7 Let $\mu$ and $\nu$ be two GT's on $\mathrm{X}$. For any $x \in \mathrm{X},\{x\}$ is $\mu$-closed or uvg-open. 
Proof. Suppose that $\{x\}$ is not $\mu$-closed. Then $X \backslash\{x\}$ is not $\mu$-open. Then either there does not exist any $\mu$-open set containing $X \backslash\{x\}$ or the only $\mu$-open set containing $X \backslash\{x\}$ is $X$ itself. Therefore, $c_{v}(X \backslash\{x\}) \subseteq X$ and hence, $X \backslash\{x\}$ is $\mu \nu g$-closed. Thus $\{x\}$ is $\mu \nu g$-open.

\section{$3(\mu, v)$-regular space and $(\mu, v)$-normal space}

Definition 4 Let $\mu$ and $v$ be two GT's on $\mathrm{X}$. Then $(\mathrm{X}, \mu, v)$ is said to be $(\mu, v)$-regular if for each $\mu$-closed set $\mathrm{F}$ of $\mathrm{X}$ not containing $\mathrm{X}$, there exist disjoint $\mathrm{V}$-open sets $\mathrm{U}$ and $\mathrm{V}$ such that $\mathrm{x} \in \mathrm{U}$ and $\mathrm{F} \subseteq \mathrm{V}$.

Theorem 8 Let $\mu$ and $\nu$ be two GT's on $\mathrm{X}$. Then the followings are equivalent:

(i) $\mathrm{X}$ is $(\mu, v)$-regular.

(ii) For each $\mathrm{x} \in \mathrm{X}$ and each $\mathrm{U} \in \mu$ containing $\mathrm{x}$ there exists $\mathrm{V} \in \boldsymbol{v}$ containing $\mathrm{x}$ such that $\mathrm{x} \in \mathrm{V} \subseteq \mathrm{c}_{\gamma}(\mathrm{V}) \subseteq \mathrm{U}$.

(iii) For each $\mu$-closed set $\mathrm{F}$ of $\mathrm{X}, \cap\left\{\mathbf{c}_{\mathrm{v}}(\mathrm{V}): \mathrm{F} \subseteq \mathrm{V} \in \mathrm{v}\right\}=\mathrm{F}$.

(iv) For each subset $\mathrm{A}$ of $\mathrm{X}$ and each $\mathrm{U} \in \mu$ with $\mathrm{A} \cap \mathrm{U} \neq \varnothing$, there exists a $\mathrm{V} \in \mathrm{v}$ such that $\mathrm{A} \cap \mathrm{V} \neq \varnothing$ and $\mathrm{c}_{\mathrm{v}}(\mathrm{V}) \subseteq \mathrm{U}$.

(v) For each non-empty subset $\mathrm{A}$ of $\mathrm{X}$ and each $\mu$-closed subset $\mathrm{F}$ of $\mathrm{X}$ with $\mathrm{A} \cap \mathrm{F}=\varnothing$, there exist $\mathrm{V}, \mathrm{W} \in \mathrm{V}$ such that $\mathrm{A} \cap \mathrm{V} \neq \varnothing, \mathrm{F} \subseteq \mathrm{W}$ and $\mathrm{W} \cap \mathrm{V}=\varnothing$.

(vi) For each $\mu$-closed set $\mathrm{F}$ with $\mathrm{x} \notin \mathrm{F}$ there exists $\mathrm{U} \in \mu$ and a $\mu$ vg-open set $\mathrm{V}$ such that $\mathrm{x} \in \mathrm{U}, \mathrm{F} \subseteq \mathrm{V}$ and $\mathrm{U} \cap \mathrm{V}=\varnothing$.

(vii) For each $\mathrm{A} \subseteq \mathrm{X}$ and each $\mu$-closed set $\mathrm{F}$ with $\mathrm{A} \cap \mathrm{F}=\varnothing$ there exists $\mathrm{U} \in \mu$ and $a \mu \nu \mathrm{g}$-open set $\mathrm{V}$ such that $\mathrm{A} \cap \mathrm{U} \neq \varnothing, \mathrm{F} \subseteq \mathrm{V}$ and $\mathrm{U} \cap \mathrm{V}=\varnothing$.

(viii) For each $\mu$-closed set $\mathrm{F}$ of $\mathrm{X}, \mathrm{F}=\cap\left\{\mathrm{c}_{\gamma}(\mathrm{V}): \mathrm{F} \subseteq \mathrm{V}, \mathrm{V}\right.$ is $\mu$ vg-open $\}$.

Proof. (i) $\Rightarrow$ (ii): Let $U$ be a $\mu$-open set containing $x$. Then $x \notin X \backslash U$, where $\mathrm{X} \backslash \mathrm{U}$ is $\mu$-closed. Then by (i) there exist $\mathrm{G}, V \in v$ such that $\mathrm{X} \backslash \mathrm{U} \subseteq \mathrm{G}$ and $x \in \mathrm{V}$ and $\mathrm{G} \cap \mathrm{V}=\varnothing$. Thus $\mathrm{V} \subseteq \mathrm{X} \backslash \mathrm{G}$ and so $\mathrm{x} \in \mathrm{V} \subseteq \mathrm{c}_{\gamma}(\mathrm{V}) \subseteq \mathrm{X} \backslash \mathrm{G} \subseteq \mathrm{U}$.

(ii) $\Rightarrow$ (iii): Let $X \backslash F \in \mu$ be such that $x \notin F$. Then by (ii) there exists $U \in v$ containing $x$ such that $x \in \mathrm{U} \subseteq \mathrm{c}_{v}(\mathrm{U}) \subseteq X \backslash F$. So, $\mathrm{F} \subseteq X \backslash \mathrm{c}_{v}(\mathrm{U})=\mathrm{V}$ (say) $\in v$ and $\mathrm{U} \cap \mathrm{V}=\varnothing$. Thus $\mathrm{x} \notin \mathrm{c}_{v}(\mathrm{~V})$. Thus $\mathrm{F} \supseteq \cap\left\{\mathrm{c}_{v}(\mathrm{~V}): \mathrm{F} \subseteq \mathrm{V} \in \mathrm{v}\right\}$.

(iii) $\Rightarrow$ (iv): Let $U \in \mu$ with $x \in U \cap A$. Then $x \notin X \backslash U$ and hence by (iii) there exists a $v$-open set $W$ such that $X \backslash U \subseteq W$ and $x \notin \mathrm{c}_{v}(W)$. We put 
$V=X \backslash c_{v}(W)$, which is a $v$-open set containing $x$ and hence $A \cap V \neq \varnothing$ (as $x \in A \cap \mathrm{V})$. Now $\mathrm{V} \subseteq X \backslash W$ and so $c_{v}(\mathrm{~V}) \subseteq X \backslash W \subseteq \mathrm{U}$.

(iv) $\Rightarrow(v)$ : Let $F$ be a $\mu$-closed set as in the hypothesis of $(v)$. Then $X \backslash F$ is a $\mu$-open set and $(X \backslash F) \cap A \neq \varnothing$. Then there exists $V \in v$ such that $A \cap V \neq \varnothing$ and $c_{v}(V) \subseteq X \backslash F$. If we put $W=X \backslash c_{v}(V)$, then $F \subseteq W$ and $W \cap V=\varnothing$.

$(\mathrm{v}) \Rightarrow(\mathrm{i})$ : Let $\mathrm{F}$ be a $\mu$-closed set not containing $x$. Then by $(\mathrm{v})$, there exist $\mathrm{W}, \mathrm{V} \in \mathrm{v}$ such that $\mathrm{F} \subseteq \mathrm{W}$ and $\mathrm{x} \in \mathrm{V}$ and $\mathrm{W} \cap \mathrm{V}=\varnothing$.

(i) $\Rightarrow$ (vi): Obvious as every $v$-open set is $\mu \nu$-open (by Proposition 1 ).

(vi) $\Rightarrow$ (vii): Let $F$ be a $\mu$-closed set such that $A \cap F=\varnothing$ for any subset $A$ of $X$. Thus for $a \in A, a \notin F$ and hence by (vi), there exists $U \in \mu$ and a $\mu v g$-open set $\mathrm{V}$ such that $\mathrm{a} \in \mathrm{U}, \mathrm{F} \subseteq \mathrm{V}$ and $\mathrm{U} \cap \mathrm{V}=\varnothing$ and $\mathrm{A} \cap \mathrm{U} \neq \varnothing$.

(vii) $\Rightarrow$ (i): Let $x \notin F$, where $F$ be $\mu$-closed. Since $\{x\} \cap F=\varnothing$, by (vii) there exist $\mathrm{U} \in \mu$ and a $\mu v$ g-open set $W$ such that $x \in U, F \subseteq W$ and $\mathrm{U} \cap \mathrm{W}=\varnothing$. Now put $\mathrm{V}=i_{v}(\mathrm{~W})$. Then $\mathrm{F} \subseteq \mathrm{V}$ (by Theorem 4) and $\mathrm{U} \cap \mathrm{V}=\varnothing$.

(iii) $\Rightarrow$ (viii): We have $\mathrm{F} \subseteq \cap\left\{\mathbf{c}_{v}(\mathrm{~V}): \mathrm{F} \subseteq \mathrm{V}\right.$ and $\mathrm{V}$ is $\mu \nu$ g-open $\} \subseteq \cap\left\{\mathrm{c}_{\gamma}(\mathrm{V})\right.$ : $\mathrm{F} \subseteq \mathrm{V}$ and $\mathrm{V}$ is $\mathrm{V}$-open $\}=\mathrm{F}$.

(viii) $\Rightarrow$ (i): Let $F$ be a $\mu$-closed set in $X$ not containing $x$. Then by (viii) there exists a $\mu \nu$-open set $W$ such that $F \subseteq W$ and $x \in X \backslash c_{v}(W)$. Since $F$ is $\mu$-closed and $W$ is $\mu \nu$ g-open, $F \subseteq i_{v}(W)$ (by Theorem 4). Take $V=i_{v}(W)$. Then $\mathrm{F} \subseteq \mathrm{V}, \mathrm{x} \in \mathrm{X} \backslash \mathrm{c}_{\mathrm{V}}(\mathrm{V})=\mathrm{U}$ (say) (as $(\mathrm{X} \backslash \mathrm{F}) \cap \mathrm{V}=\varnothing$ ) and $\mathrm{U} \cap \mathrm{V}=\varnothing$.

Definition 5 Let $\mu$ and $\nu$ be two GT's on a set $\mathrm{X}$. Then $(\mathrm{X}, \mu, \nu)$ is said to be $(\mu, v)$-normal if for disjoint $\mu$-closed sets $\mathrm{F}_{1}$ and $\mathrm{F}_{2}$, there exist $\mathrm{U}_{1}, \mathrm{U}_{2} \in v$ such that $\mathrm{F}_{1} \subseteq \mathrm{U}_{1}, \mathrm{~F}_{2} \subseteq \mathrm{U}_{2}$ with $\mathrm{U}_{1} \cap \mathrm{U}_{2}=\varnothing$.

Theorem 9 Let $\mu$ and $\nu$ be two GT's on $\mathrm{X}$. Then the following properties are equivalent:

(i) $(X, \mu, v)$ is $(\mu, v)$-normal;

(ii) for any two disjoint $\mu$-closed sets $F_{1}, F_{2}$, there exist $\mu \vee g$-open sets $V_{1}, V_{2}$ such that $\mathrm{F}_{1} \subseteq \mathrm{V}_{1}, \mathrm{~F}_{2} \subseteq \mathrm{V}_{2}$ and $\mathrm{V}_{1} \cap \mathrm{V}_{2}=\varnothing$;

(iii) for any $\mu$-closed set $\mathrm{F}$ and any $\mu$-open set $\mathrm{U}$ containing $\mathrm{F}$, there exists a $\mu v g$-open set $\mathrm{V}$ such that $\mathrm{F} \subseteq \mathrm{V} \subseteq \mathrm{c}_{v}(\mathrm{~V}) \subseteq \mathrm{U}$;

(iv) for any $\mu$-closed set $\mathrm{F}$ and any $\mu$-open set $\mathrm{U}$ containing $\mathrm{F}$, there exists a v-open set $\mathrm{G}$ such that $\mathrm{F} \subseteq \mathrm{G} \subseteq \mathrm{c}_{v}(\mathrm{G}) \subseteq \mathrm{U}$;

(v) for any disjoint $\mu$-closed sets $\mathrm{F}_{1}, \mathrm{~F}_{2}$, there exists a $\mu \mathrm{vg}$-open set $\mathrm{V}$ such that $\mathrm{F}_{1} \subseteq \mathrm{V}$ and $\mathrm{c}_{v}(\mathrm{~V}) \cap \mathrm{F}_{2}=\varnothing$;

(vi) for any disjoint $\mu$-closed sets $\mathrm{F}_{1}, \mathrm{~F}_{2}$, there exists a $v$-open set $\mathrm{G}$ such that $\mathrm{F}_{1} \subseteq \mathrm{G}$ and $\mathrm{c}_{v}(\mathrm{G}) \cap \mathrm{F}_{2}=\varnothing$. 
Proof. (i) $\Rightarrow$ (ii): Follows from (i) as, every $\nu$-open set is $\mu \nu$ g-open.

(ii) $\Rightarrow$ (iii): Let $F$ be a $\mu$-closed set and $U$ be any $\mu$-open set containing $F$. Then $F$ and $X \backslash U$ are disjoint $\mu$-closed sets and by (ii) there exist $\mu \nu g$-open sets $\mathrm{V}_{1}, \mathrm{~V}_{2}$ such that $\mathrm{F} \subseteq \mathrm{V}_{1}, \mathrm{X} \backslash \mathrm{U} \subseteq \mathrm{V}_{2}$ with $\mathrm{V}_{1} \cap \mathrm{V}_{2}=\varnothing$. Since $\mathrm{V}_{2}$ is $\mu v \mathrm{v}$ open, by Theorem 4, $X \backslash U \subseteq i_{v}\left(V_{2}\right)$. Hence, $c_{v}\left(V_{1}\right) \cap i_{v}\left(V_{2}\right)=\varnothing$. Therefore, we obtain $\mathrm{F} \subseteq \mathrm{V}_{1} \subseteq \mathrm{c}_{v}\left(\mathrm{~V}_{1}\right) \subseteq \mathrm{X} \backslash \mathrm{i}_{v}\left(\mathrm{~V}_{2}\right) \subseteq \mathrm{U}$. Put $\mathrm{V}=\mathrm{V}_{1}$, then we obtain $\mathrm{F} \subseteq \mathrm{V} \subseteq \mathrm{c}_{v}(\mathrm{~V}) \subseteq \mathrm{U}$.

(iii) $\Rightarrow$ (iv): Let $F$ be a $\mu$-closed set and $U$ be any $\mu$-open set containing $F$. Then by (iii) there exists a $\mu \nu$ g-open set $\mathrm{V}$ such that $\mathrm{F} \subseteq \mathrm{V} \subseteq \mathrm{c}_{v}(\mathrm{~V}) \subseteq \mathrm{U}$. By Theorem $4, F \subseteq i_{v}(V)$. Put $G=i_{v}(V)$. Then $G$ is a $v$-open set. Furthermore, we obtain $\mathrm{F} \subseteq \mathrm{G} \subseteq \mathrm{c}_{v}(\mathrm{G}) \subseteq \mathrm{c}_{v}(\mathrm{~V}) \subseteq \mathrm{U}$.

(iv) $\Rightarrow(v)$ : Let $F_{1}, F_{2}$ be any two disjoint $\mu$-closed sets. Since $X \backslash F_{2}$ is a $\mu$-open set containing $F_{1}$, by (iv) there exists a $V$-open set $V$ such that $\mathrm{F}_{1} \subseteq \mathrm{V} \subseteq \mathrm{c}_{v}(\mathrm{~V}) \subseteq \mathrm{X} \backslash \mathrm{F}_{2}$. By Proposition $1, \mathrm{~V}$ is $\mu \nu \mathrm{g}$-open. Furthermore, we have $\mathrm{F}_{1} \subseteq \mathrm{V}$ and $\mathrm{c}_{v}(\mathrm{~V}) \cap \mathrm{F}_{2}=\varnothing$.

$(\mathrm{v}) \Rightarrow(\mathrm{vi})$ : Let $\mathrm{F}_{1}, \mathrm{~F}_{2}$ be any disjoint $\mu$-closed sets. Then there exists a $\mu \nu \mathrm{g}$ open set $\mathrm{V}$ such that $\mathrm{F}_{1} \subseteq \mathrm{V}$ and $\mathrm{c}_{v}(\mathrm{~V}) \cap \mathrm{F}_{2}=\varnothing$. By Theorem $4, \mathrm{~F}_{1} \subseteq \mathrm{i}_{v}(\mathrm{~V})$. Set $G=i_{v}(V)$. Then $G \in v, F_{1} \subseteq G$ and $c_{v}(G) \cap F_{2}=\varnothing$.

(vi) $\Rightarrow$ (i): Let $F_{1}, F_{2}$ be any two disjoint $\mu$-closed sets. Then by (vi) there exists $G \in v$ such that $F_{1} \subseteq G$ and $c_{v}(G) \cap F_{2}=\varnothing$. Now, put $U_{1}=G$ and $\mathrm{U}_{2}=\mathrm{X} \backslash \mathrm{c}_{v}(\mathrm{G})$. Then $\mathrm{U}_{1}$ and $\mathrm{U}_{2}$ are disjoint $v$-open sets, $\mathrm{F}_{1} \subseteq \mathrm{U}_{1}$ and $\mathrm{F}_{2} \subseteq \mathrm{U}_{2}$. This shows that $(X, \mu, v)$ is $(\mu, v)$-normal.

\section{Conclusion}

Interchanging $\mu$ and $\nu$ by different weak forms of open sets we can characterize different weak forms of generalized open sets and different weak forms of regular and normal spaces. If $\mu=\nu$, then we get the results obtained in [6].

\section{Acknowledgment}

The author acknowledges the financial support from UGC, New Delhi.

\section{References}

[1] Á. Császár, Generalized topology, generalized continuity, Acta Math. Hungar., 96 (2002), 351-357. 
[2] Á. Császár, Generalized open sets in generalized topologies, Acta Math. Hungar., 106 (2005), 53-66.

[3] Á. Császár, Remarks on quasi topologies, Acta Math. Hungar., 119 (2008), 197-200.

[4] B. Roy, T. Noiri, Unifiation of $\lambda$-closed sets via generalized topologies, Novi Sad J. Math., 43 (1), (2013), 51-58.

[5] E. Ekici, B. Roy, New generalized topologies on generalized topological spaces due to Császár, Acta Math. Hungar., 132 (2011), 117-124.

[6] B. Roy, On a type of generalized open sets, Applied Gen. Topol., 12 (2)(2011), 16-173.

Received: May 25, 2015 